\title{
Effects of PHA-665752 and vemurafenib combination treatment on in vitro and murine xenograft growth of human colorectal cancer cells with $\mathrm{BRAF}^{\mathrm{V} 600 \mathrm{E}}$ mutations
}

\author{
JIE ZHI ${ }^{1}$, ZHONGXIN LI ${ }^{2}$, JIAN LV ${ }^{2}$, BO FENG ${ }^{1}$, DONGHAI YANG ${ }^{2,3}$, LIANG XUE $^{2,4}$, \\ ZHAOLONG ZHAO $^{2}$, YANNI ZHANG ${ }^{2}$, JIANHUA WU ${ }^{5}$, YINGCHAO JV ${ }^{5}$ and YITAO JIA ${ }^{1}$
}

${ }^{1}$ Department of Oncology, Hebei General Hospital, Shijiazhuang, Hebei 050051; ${ }^{2}$ Second Department of Surgery, The Fourth Hospital of Hebei Medical University, Shijiazhuang, Hebei 050011; ${ }^{3}$ Department of Emergency Medicine, The Second Affiliated Hospital of Xingtai Medical College, Xingtai, Hebei 054000; ${ }^{4}$ Department of Surgery, The Affiliated Hospital of Hebei University of Engineering, Handan, Hebei 056038; ${ }^{5}$ Experimental Animal Center, The Fourth Hospital of Hebei Medical University, Shijiazhuang, Hebei 050011, P.R. China

Received May 14, 2017; Accepted November 6, 2017

DOI: $10.3892 / 01.2018 .7770$

\begin{abstract}
It remains unknown whether blockade of B-Raf proto-oncogene, serine/threonine kinase (BRAF) ${ }^{\mathrm{V} 600 \mathrm{E}}$ signaling and MET proto-oncogene, receptor tyrosine kinase (c-Met) signaling is effective in suppressing the growth of human colorectal cancer (CRC) cells. The present study investigated the effects of the vemurafenib alone and in combination with c-Met inhibitor PHA-665752 on the growth of human CRC cells in vitro and in mouse xenografts. HT-29 and RKO CRC cell lines with $\mathrm{BRAF}^{\mathrm{V} 600 \mathrm{E}}$ mutations and mice bearing HT-29 xenografts were treated with vemurafenib in the absence or presence of PHA-665752. Cell viability and cycle phase were respectively examined by using the MTT and flow cytometry assay. Immunohistochemistry was conducted to detect the protein expression levels of hepatocyte growth factor (HGF), phosphorylated (p)-c-Met, p-AKT serine/threonine kinase (AKT) and p-extracellular signal-regulated kinase (p-ERK). The MTT assay demonstrated that the growth of RKO and HT-29 cells was inhibited by PHA- 665752 in a time- and dose-dependent manner $(\mathrm{P}<0.05)$, however no significant suppressive effects were observed with vemurafenib. Relative to the PHA-665752 or vemurafenib stand-alone treatment groups, the combination of PHA-665752 and vemurafenib had a significant inhibitory effect on the proliferation of CRC cell lines $(\mathrm{P}<0.05)$. The mean tumor volume in mice treated with vemurafenib in combination with PHA-665752 was significantly smaller compared with those treated with only
\end{abstract}

Correspondence to: Professor Yitao Jia, Department of Oncology, Hebei General Hospital, 348 West Heping Road Shijiazhuang, Hebei 050051, P.R. China

E-mail: jiayitao99@163.com

Key words: colorectal cancer, PHA-665752, vemurafenib, BRAF, c-Met vemurafenib or PHA-665752 ( $\mathrm{P}<0.05)$. Flow cytometry assay revealed that the G0/G1 phase frequency was significantly increased in the combination group compared with any other treatment groups $(\mathrm{P}<0.05)$. Immunohistochemistry demonstrated that vemurafenib in combination with PHA-665752 effectively induced the expression of p-c-Met, p-AKT and p-ERK, however had no effect on HGF.

\section{Introduction}

Colorectal cancer (CRC) is one of the principal causes of cancer worldwide (1). Unfortunately, even if a 5-year survival prognosis can be given to $90 \%$ of patients in the early stages, disappointing treatment outcomes are recorded in subjects with extensive local invasion or distant metastases. Generally, their 5-year survival rate is less than $15 \%$ (2) and, even if suitable for receiving adjuvant chemotherapy, disease-related deaths remain stubbornly high.

A recent steady stream of important breakthroughs has dramatically improved our understanding of CRC. One such discovery identified the BRAF mutation as common in metastatic CRC patients, especially those with a right-side colon cancer and a poorly differentiated tumor (3). Vemurafenib is a potent and selective inhibitor of mutated BRAF. Chapman et al (4) revealed that vemurafenib achieved $40 \%$ response rates in melanoma with a BRAF mutation. However, unlike melanoma, the effect of vemurafenib in CRC patients with a BRAF mutation is often negligible, resulting in a clinical response in only $5 \%$ of patients (5). This discrepancy of outcomes suggests that different cancer types may present important variations even if they share the same $\mathrm{BRAF}^{\mathrm{V} 600 \mathrm{E}}$ mutation.

Growing evidence has revealed that resistance to $\mathrm{BRAF}^{\mathrm{V} 600 \mathrm{E}}$ inhibition depends in part on altered c-Met signaling in cancers. Blocking c-Met signaling may therefore help reverse resistance to vemurafenib in $\mathrm{BRAF}^{\mathrm{V} 600 \mathrm{E}}$-targeted therapy $(6,7)$. Byeon et al (6) have reported that dual inhibition of $\mathrm{BRAF}^{\mathrm{V} 600 \mathrm{E}}$ and c-Met leads to a reversal of the epithelial-to-mesenchymal 
transition, resulting in decreased resistance to chemotherapy and a positive therapeutic response in thyroid cancer. To date, only a few studies have focused on the effects of c-Met inhibitors and anti-BRAF agents as a combined treatment option for CRCs. We therefore investigated the combined effect of vemurafenib and PHA-665752, a c-Met inhibitor, on in vitro and in vivo growth of human CRC cells, with the goal of identifying suitable clinical combinations.

\section{Materials and methods}

Animals. Fifty-six female BALB/c nu/nu nude mice (age, 4-5 weeks; weight, $20 \mathrm{~g}$ each) were purchased from HFK Bioscience Co., Ltd. (Beijing, China) and raised under specific pathogen-free conditions at the Animal Experimental Center of the Fourth Hospital of Hebei Medical University (Shijiazhuang, China). The animals were allowed to adapt to the housing conditions for 5 days before being used in experiments. The experimental protocol pertaining to the animal study was approved by the Institutional Animal Care and the Ethics Committee of the Fourth Hospital of Hebei Medical University In addition, animal studies were conducted in accordance with the National Institutes of Health Guidelines for the Care and Use of Laboratory Animals.

Cell lines and antibodies. Human CRC cell lines RKO and HT-29 (BRAF ${ }^{\mathrm{V} 600 \mathrm{E}}$ mutant) were acquired from the Cell Bank of the Type Culture Collection of the Chinese Academy of Sciences (Shanghai, China). HT-29 cells were cultured in McCoy's 5A medium and RKO cells were cultured in MEM (both from Gibco; Thermo Fisher Scientific, Inc., Waltham, MA, USA). Cells were maintained in the presence of $100 \mathrm{U} / \mathrm{ml}$ penicillin and $100 \mu \mathrm{g} / \mathrm{ml}$ streptomycin at $37^{\circ} \mathrm{C}$ in an atmosphere containing $5 \% \mathrm{CO}_{2}$. The medium was supplemented with $10 \%$ fetal calf serum (FBS; PAN-Biotech $\mathrm{GmbH}$, Aidenbach, Germany).

The following rabbit polyclonal antibodies were purchased from Bioworld Technology (Louis Park, MN, USA): Anti-phosphorylated (p)-c-Met (BS4752), anti-AKT (BS1007), anti-p-AKT (BS4007), and anti-p-extracellular signal-regulated kinase (anti-p-ERK) (BS5016). An anti-hepatocyte growth factor (HGF) rabbit polyclonal antibody (BS1025R) was purchased from Bioss Inc. (Woburn, MA, USA). Vemurafenib was purchased from Cayman Chemical Co. (Ann Arbor, MI, USA); PHA-665752 was purchased from Selleck Chemicals LLC (Houston, TX, USA).

\section{3-(4,5-Dimethylthiazol-2-yl)-2,5-diphenyltetrazolium (MTT)} assay. Cells were plated on 96-well microtiter plates at a density of $3 \times 10^{3}$ cells/well, and cultured overnight to allow for cell attachment. Cells were treated with PHA-665752 (1, 1.5, $2,2.5,3,3.5$, or $4 \mu \mathrm{mol} / 1)$ and/or vemurafenib $(0.01,0.1,0.5,1$, $3,6$, or $9 \mu \mathrm{mol} / \mathrm{l})$. Each microtiter plate was incubated for $4 \mathrm{~h}$ at $37^{\circ} \mathrm{C}$. The half-maximal inhibitory concentration $\left(\mathrm{IC}_{50}\right)$ of PHA-665752 was calculated as follows:

Percent inhibition $=\left[1-\frac{\text { Mean absorbance of experiment al wells }- \text { Mean absorbance of blank wells }}{\text { Mean absorbance of control wells }- \text { Mean absorbance of blank wells }}\right] \times 100 \%$

The $\mathrm{IC}_{50}$ was calculated when it was equal to $50 \%$. In single-drug treatment experiments, a suitable $\mathrm{IC}_{50}$ value for vemurafenib could not be obtained because of low response to the drug. Thus, in the combined treatment experiment, we used a fixed concentration of PHA-665752 and a varying concentration of vemurafenib. The MTT assay determined the viability of CRC cells at 24,48 , and 72 h. A Thermo Plate microplate reader (Thermo Fisher Scientific, Inc.) was used to measure sample absorbance at $490 \mathrm{~nm}$.

Flow cytometry. For cell cycle detection, cells were seeded in 6-well plates at a density of $12 \times 10^{4}$ cells/well for HT-29 and $9 \times 10^{4}$ cells/well for RKO cells. Cells were washed three times with cold phosphate-buffered saline (PBS) and then stained with $50 \mu 1$ propidium oxide (Sigma-Aldrich, St. Louis, MO, USA) for $30 \mathrm{~min}$. Each sample was harvested at log phase and fixed in $70 \%$ ethanol at $4^{\circ} \mathrm{C}$ overnight in the dark. Quantification of cell cycle distribution was performed with a FACScan system (Beckman Coulter, Inc., Brea, CA, USA). The percentage of cells in G0/G1, S, and G2-M phases was calculated and compared.

Mouse xenograft studies. Cancer cells $\left(5.5 \times 10^{7}\right)$ were subcutaneously implanted into the left abdominal region of mice. Both PHA-665752 and vemurafenib were prepared by dilution in dimethyl sulfoxide (DMSO). Treatments were administered when tumor length reached $\sim 0.5-0.7 \mathrm{~cm}$. Mice were divided into four groups ( $\mathrm{n}=10 /$ group): vemurafenib group $[75 \mathrm{mg} / \mathrm{kg}$ peroral (p.o.) twice a day, interval time $\geq 8$ h], PHA-665752 group [25 mg/kg intraperitoneal (i.p.) every other day], combined group (vemurafenib $75 \mathrm{mg} / \mathrm{kg}$ p.o. twice a day, interval time $\geq 8 \mathrm{~h}$; PHA-665752 $25 \mathrm{mg} / \mathrm{kg}$ i.p. every other day), and DMSO control group (2.5\% DMSO $200 \mu$ l i.p. every other day). Tumor volume was measured every 3 days, and calculated using the following formula:

Tumor volume $=\left[\right.$ large diameter of tumor $\left.\mathrm{x}(\text { small diameter of tumor })^{2}\right] / 2$

The treatment lasted for 3 weeks. Then, mice were sacrificed under anesthesia, and tumor tissue was obtained and fixed in $10 \%$ formaldehyde solution prior to immunohistochemical analysis.

Immunohistochemical analysis. Samples were fixed by formalin, embedded in paraffin, and cut into sections (4-6- $\mu \mathrm{m}$ thick). Immunochemical staining (IHC) was performed using the Envision plus detection system (Dako, Glostrup, Denmark) according to the manufacturer's instructions. Sections were incubated with methanol/hydroperoxide (9:1) for $20 \mathrm{~min}$ at room temperature to block endogenous peroxidase activity, and washed with PBS. Sections were incubated at $4^{\circ} \mathrm{C}$ overnight with primary antibodies against HGF (1:100), p-c-Met (1:100), p-Akt (1:200), and p-ERK (1:200). Secondary antibodies (Zhongshan Golden Bridge Biotechnology Co., Ltd., Beijing, China) were added, sections were incubated at $37^{\circ} \mathrm{C}$ for $45 \mathrm{~min}$, and washed with PBS. DAB staining was used for coloring sections and nuclei were counterstained with hematoxylin. Yellow, yellowish-brown, or darker immunohistochemical staining was considered as positive. The density of positive staining was measured using a computerized image system composed of a charge-coupled device camera (DFC420; Leica Microsystems Imaging Solution, Ltd., Cambridge, UK) connected to a microscope (DM IRE2; 
A

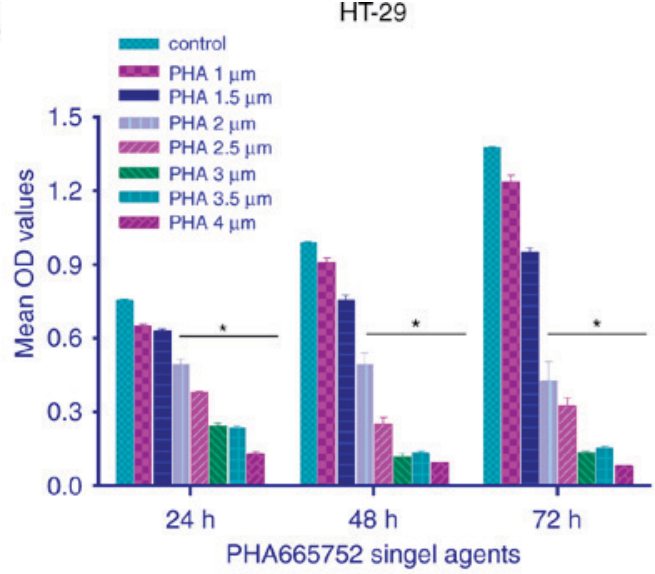

C

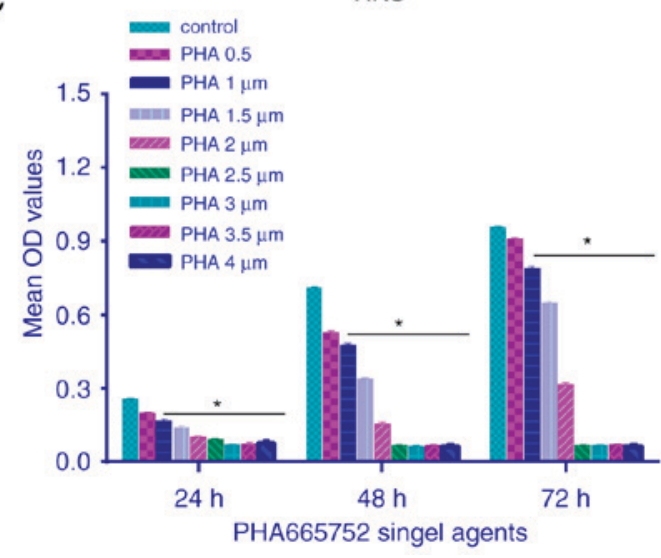

B

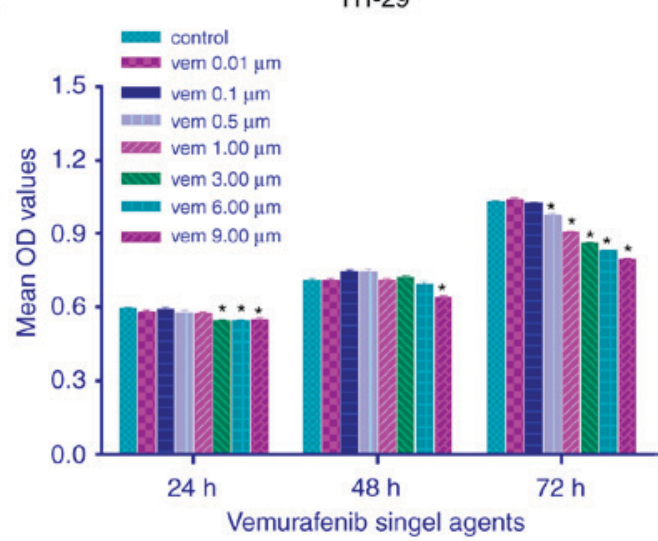

D

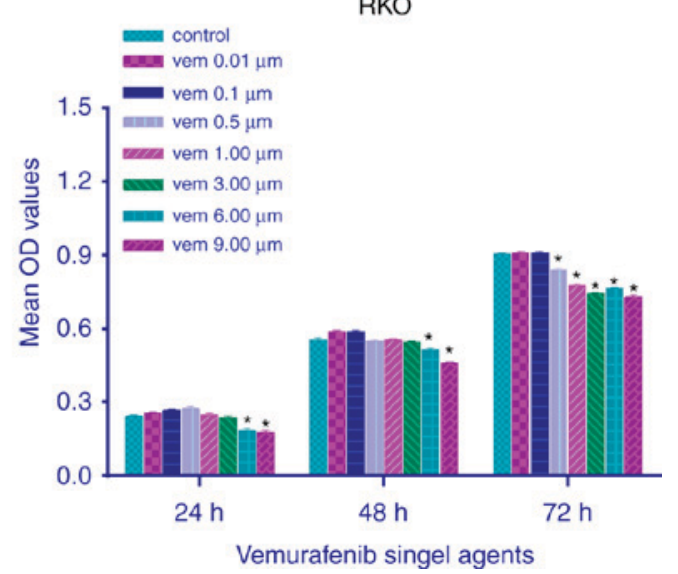

Figure 1. Effect of different concentrations (in $\mu \mathrm{M}$ given as standard symbols) of PHA-665752 and vemurafenib on cell proliferation in (A and B) HT-29 and (C and D) RKO cell lines. In vitro sensitivity of CRC cells to PHA-665752 and vemurafenib was determined using the MTT assay. Mean OD was used to detect cell viability following treatment with different concentrations of drugs for 24,48 and $72 \mathrm{~h}$. Values correspond to means. Data are represented as mean \pm standard error of the mean $(n=7)$. One-way ANOVA followed by the LSD test was used for statistical analysis, "P<0.05 vs. control, separately. OD, optical density; LSD, least significant difference.

A

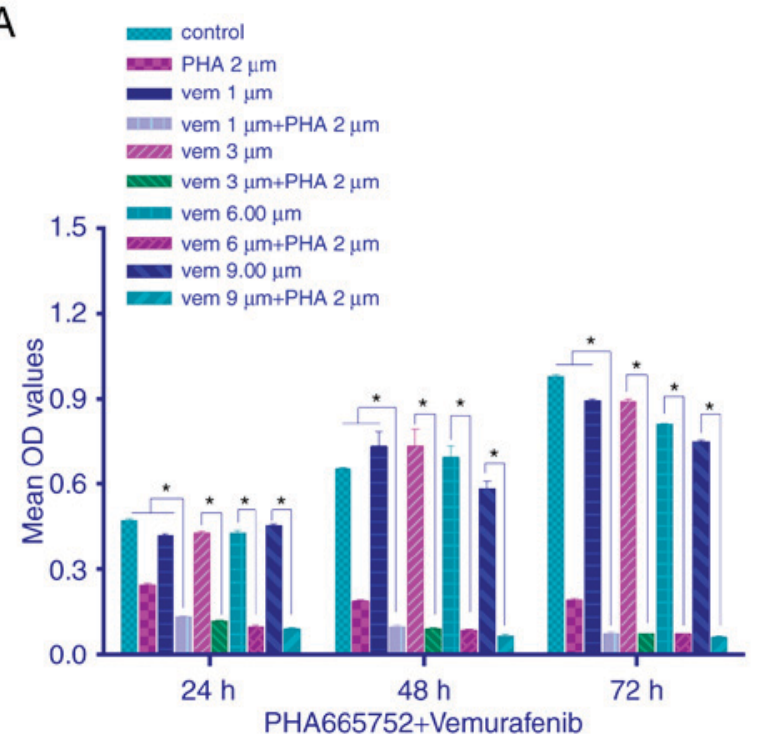

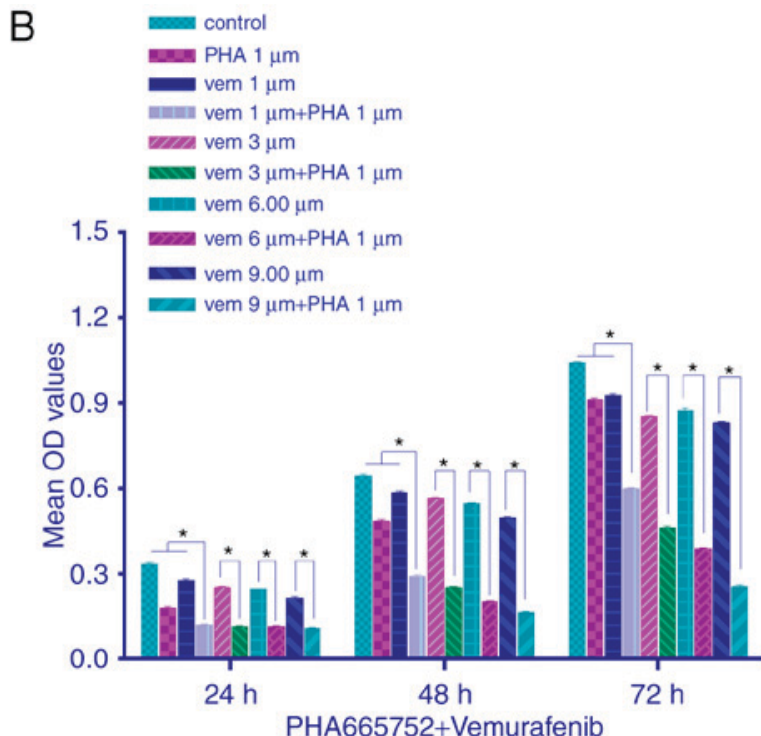

PHA665752+Vemurafenib

Figure 2. Effect of HA-665752 and vemurafenib (concentrations in $\mu \mathrm{M}$ given as symbols) on the proliferation of (A) HT-29 and (B) RKO cell lines. Drugs were applied alone or in combination for 24, 48 and $72 \mathrm{~h}$. One-way ANOVA followed by the LSD post hoc test was used for statistical analysis to compare differences between the control and treated groups. Each bar represents the mean $\pm \mathrm{SD}(\mathrm{n}=7),{ }^{*} \mathrm{P}<0.05$. LSD, least significant difference.

Leica). The mean number of immunopositive cells was determined in five fields-of-view at $\mathrm{x} 400$ magnification using Leica
Qwin Plus V3 software (Leica Microsystems GmbH, Wetzlar, Germany). 
A

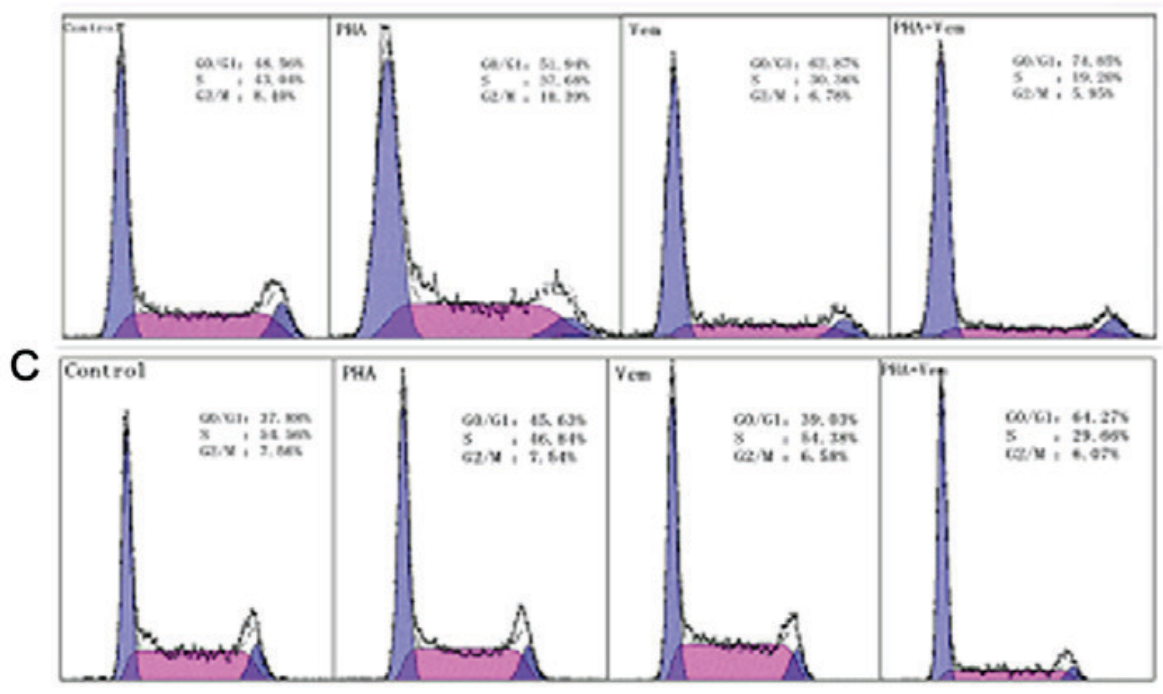

B
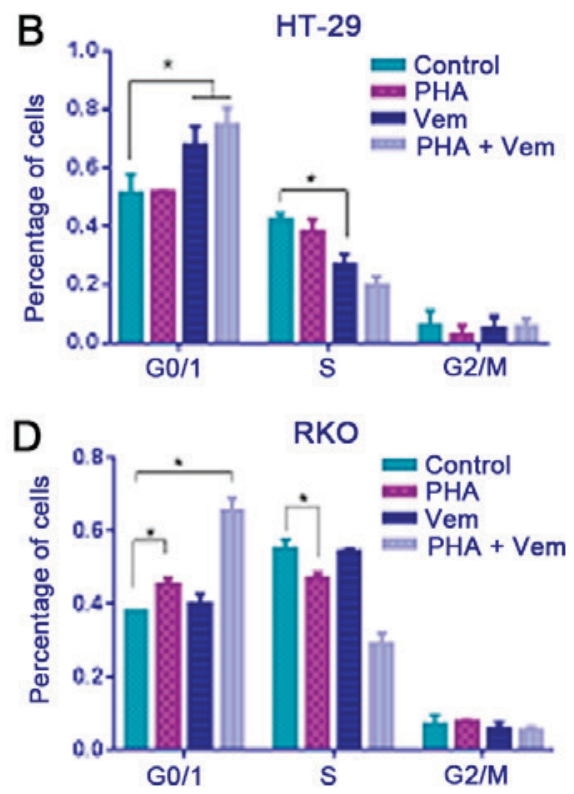

Figure 3. In vitro comparison of the effect of PHA-665752 and vemurafenib on the cell cycle. Effect of chemotherapy on cell cycle progression in (A) HT-29 and (C) RKO cells. The cycle distribution of apoptotic cells percentage in (B) HT-29 and (D) RKO cells. Tumor cells were treated with PHA-665752, vemurafenib, or a combination of the two, after which cell distribution in different phases of the cell cycle was analyzed by flow cytometry. Experiments were repeated three times. ${ }^{*} \mathrm{P}<0.05$.

Statistical analysis. Data for continuous variables are shown as mean \pm standard deviation (SD). Differences among groups were compared using Student's t-test, one-way analysis of variance (ANOVA). Statistical significance was considered at P-values $<0.05$. All data were analyzed using SPSS software (version 21.0; SPSS, Inc., Chicago, IL, USA).

\section{Results}

Growth suppression by PHA-665752 and resistance to vemurafenib in CRC cells in vitro. The effect of PHA-665752 on the viability of RKO and HT-29 cells was evaluated using the MTT assay at 24, 48, and $72 \mathrm{~h}$ (Fig. 1). PHA-665752 significantly inhibited the proliferation of CRC cells with a $\mathrm{BRAF}^{\mathrm{V} 600 \mathrm{E}}$ mutation in a time- and dose-dependent manner $(\mathrm{P}<0.05)$. In addition, $\mathrm{IC}_{50}$ values of $\mathrm{PHA}-665752$ against HT-29 and RKO cells were 2 and $1 \mu \mathrm{mol} / 1$, respectively (Fig. 1A and C). Vemurafenib slightly inhibited cell growth only at a sufficiently high concentration; consequently, we could not calculate its $\mathrm{IC}_{50}$ value (Fig. $1 \mathrm{~B}$ and $\mathrm{D} ; \mathrm{P}<0.05$ ). These findings suggested that $\mathrm{CRC}$ cells with a $\mathrm{BRAF}^{\mathrm{V} 600 \mathrm{E}}$ mutation were resistant to vemurafenib therapy, whereas the c-Met-related pathway could mediate CRC cell growth and proliferation in vitro.

Combined PHA-665752 and vemurafenib treatment is more effective than either agent alone in inhibiting CRC cell proliferation. We explored the proliferation of CRC cells by MTT assay, following a combined treatment with PHA-665752 (2 $\mu \mathrm{mol} / 1$ for HT-29 cells and $1 \mu \mathrm{mol} / 1$ for RKO cells) and vemurafenib at variable doses. Cell proliferation was inhibited $(\mathrm{P}<0.05)$ to a greater extent with the combined treatment than with any of the two drugs alone. Moreover, as the concentration of vemurafenib but not PHA-665752 increased, the inhibitory effect, too, increased (Fig. 2; $\mathrm{P}<0.05$ ). These results indicated

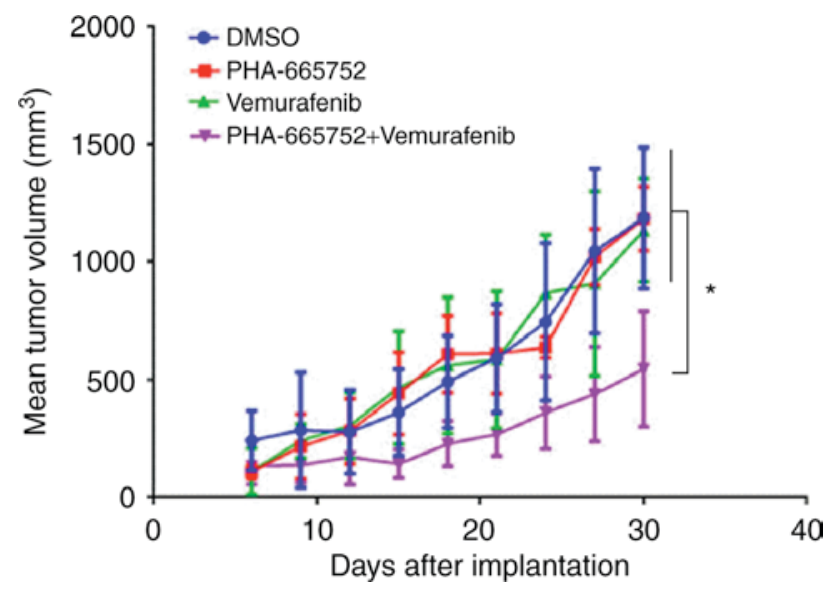

Figure 4. Tumor growth curves showing the in vivo antitumor effect of PHA-665752, vemurafenib, and a combination of the two. Tumor size was measured starting after 6 days and then once every 3 days. The LSD post hoc test was used for statistical analysis to compare differences between groups ( $\mathrm{n}=10 /$ group), ${ }^{*} \mathrm{P}<0.05$. LSD, least significant difference.

that in vitro combined therapy using PHA-665752 plus vemurafenib could significantly decrease tumor cell growth, with PHA-665752 reversing vemurafenib drug resistance.

Effect of PHA-665752, vemurafenib, and a combination of both drugs on cell cycle progression. Flow cytometry revealed that treatment with vemurafenib caused a marked increase with G0/G1 and decrease with S phase frequency in HT-29 cells compared with the control group (Figs. $3 \mathrm{~A}$ and $\mathrm{B} ; \mathrm{P}<0.05$ ). In $\mathrm{RKO}$ cells, the proportion of cells in $\mathrm{G} 0 / \mathrm{G} 1$ increased following PHA-665752 treatment $(\mathrm{P}<0.05)$, whereas that in $\mathrm{S}$ phase decreased relative to the control (Fig. 3B; $\mathrm{P}<0.05$ ). A combination of PHA-665752 and vemurafenib had a marked effect on G0/G1 phase frequency compared with the control 


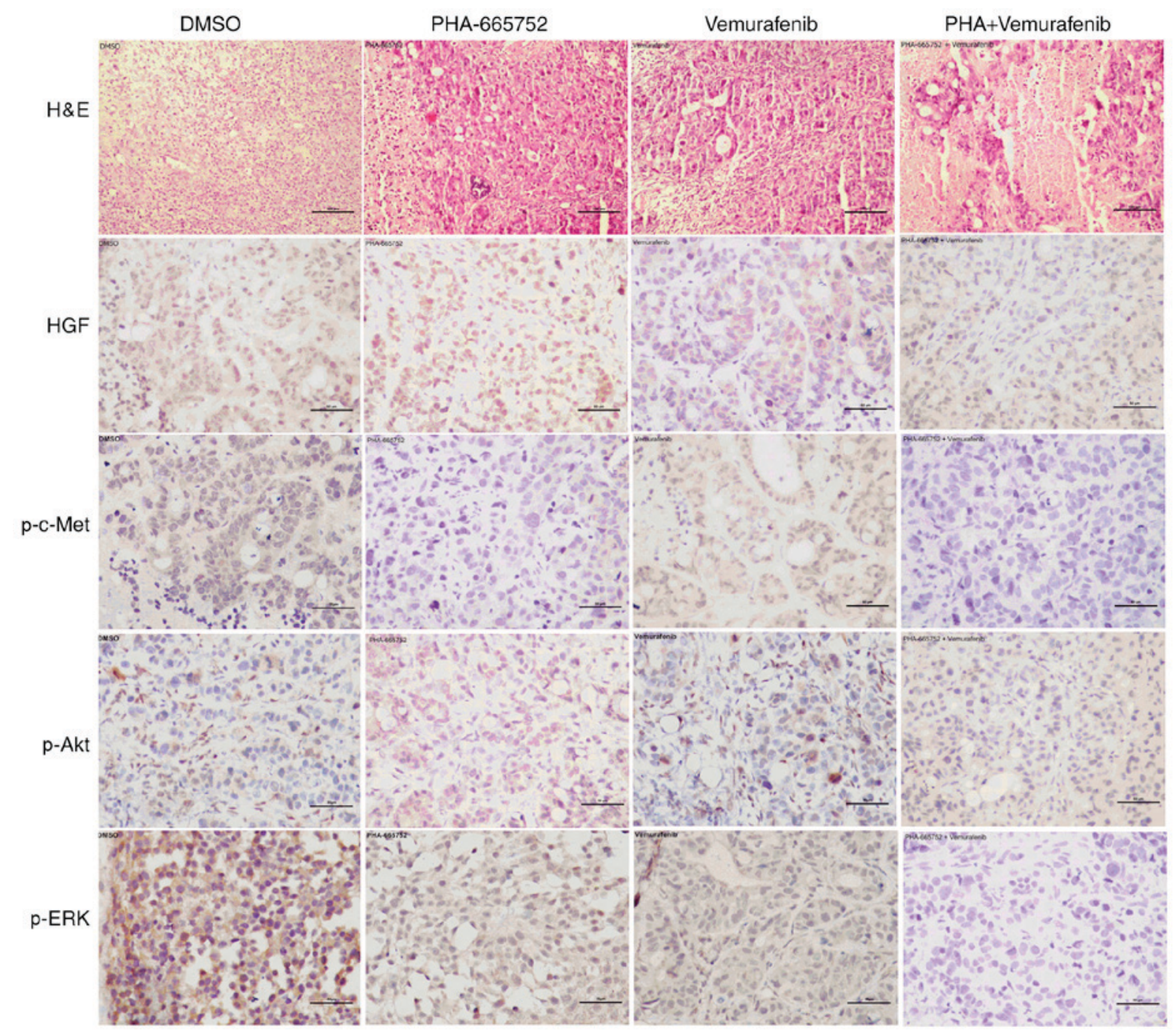

Figure 5. Immunohistochemical analysis of protein expression. Expression of phosphorylated (p)-c-Met, p-AKT, and p-ERK was high in the dimethyl sulfoxide (DMSO) control group but low in mice treated with PHA-665752 plus vemurafenib. Images are representative of immunostained tumor samples taken from the control and drug-treated groups. H\&E, scale bar, $100 \mu \mathrm{m}$; IHC, scale bar, $50 \mu \mathrm{m}$. ERK, extracellular signal-regulated kinase; c-Met, MET proto-oncogene, receptor tyrosine kinase.

in both CRC cell lines (Fig. 3C and D; P<0.05). This finding implied that HT-29 cells in G0/G1 phase were killed by vemurafemib, whereas RKO cells in G0/G1 were merely blocked by PHA-665752; their effect was significantly potentiated when the drugs were combined.

Combined PHA-665752 and vemurafenib treatment is more effective than either agent alone in suppressing growth of mouse xenografts bearing CRC cells. Thirty days after being subcutaneously implanted in the left abdominal region, tumor volumes were measured. Tumors that received combined treatment, PHA-665752 alone, vemurafenib alone, or DMSO were: $549.22 \pm 240.93,1207.25 \pm 129.53,1137.31 \pm 220.21$, $1213.85 \pm 295.34 \mathrm{~mm}^{3}$, respectively (Fig. 4). Thus, tumor volume was significantly smaller in mice subjected to combined treatment $(\mathrm{P}<0.05)$. These findings suggested that combined treatment was more effective in controlling tumor growth in mice than either PHA-665752 or vemurafenib alone.

Combined therapy with PHA-665752 and vemurafenib indicates involvement of the PI3K/AKT and MEK/ERK signaling pathway in vivo. Finally, we used immunohistochemistry to evaluate the expression of p-c-Met, p-ERK, and p-AKT following inhibition with PHA-665752 alone, vemurafenib alone, or a combination of the two. Except for HGF, the expression of the other target proteins was significantly lower in mice treated with PHA-665752 plus vemurafenib than DMSO (Fig. 5). These findings suggest that effectiveness of the combined anticancer treatment may depend on a shift of the phosphorylation status of ERK and AKT.

\section{Discussion}

BRAF is a protein directly downstream from RAS in the classical MAPK cascade (8). RAS activates RAF by recruiting RAF and simulating its dimerization. In CRC, BRAF mutations occur most commonly at the V600 site, particularly in patients with right-side colon cancer, who are more likely to present with poorly differentiated tumors $(9,10)$. Cancers with a BRAF ${ }^{\mathrm{V} 600 \mathrm{E}}$ mutation have been shown to lead to a poor prognosis (11). Therefore, anti-BRAF ${ }^{\mathrm{V} 600 \mathrm{E}}$ therapy is considered effective in oncotherapy. Vemurafenib, a selective inhibitor of $\mathrm{BRAF}^{\mathrm{V} 600 \mathrm{E}}$, 
has achieved a high objective response rate in $\sim 50 \%$ of melanomas, improving overall survival compared with traditional chemotherapy (4). In contrast to the high response rates seen in melanomas, therapeutic efficacy in CRC has been poorly characterized. In our study, we report that the c-Met inhibitor PHA-665752 could suppress CRC cell survival when used alone, whereas the $\mathrm{BRAF}^{\mathrm{V} 600 \mathrm{E}}$ inhibitor vemurafenib had no significant effect on reducing CRC cell viability. Interestingly, dual inhibition of c-Met and $\mathrm{BRAF}^{\mathrm{V} 600 \mathrm{E}}$ resulted in a significantly stronger inhibitory effect on cancer cells than either agent alone. Therefore, inhibition of BRAF signaling together with c-Met-targeted therapy may offer a new effective approach for $\mathrm{CRC}$ treatment in CRC patients with a $\mathrm{BRAF}^{\mathrm{V} 600 \mathrm{E}}$ mutation.

Along with tumor initiation, progression, and even metastases, tumor cells' viability, proliferation, and death represent important parameters. Here, cell cycle analysis indicates that $\mathrm{BRAF}^{\mathrm{V} 600 \mathrm{E}}$ and c-Met inhibitors alone can only block cell growth of RKO and HT-29 cells by inducing G0/G1 arrest. However, vemurafenib plus PHA-665752 inhibit cell proliferation of both cell lines, indicating that the antitumor effect of the combined treatment may be achieved by interrupting normal cell cycling profiles. The finding is in line with previous studies on inhibition of the cell cycle in gastric cancer and non-small cell lung cancer $(12,13)$.

In vivo, cancer develops around a solid tumor in the context of a tumor microenvironment $(14,15)$. This specific microenvironment is not only a cause but also the consequence of tumorigenesis. Tumor, mesenchymal, and nominal cells co-evolve dynamically through direct and/or indirect cellular interactions to elicit various specific biological programs $(16,17)$. The tumor microenvironment is important for tumor initiation, maintenance, and even metastasis (18); however, how and why the tumor microenvironment can contribute to cancer remains unclear (19). In our study, PHA-665752 showed significant antitumor ability in vitro, but poor inhibition of tumor volume growth in vivo. This may be due to the complex microenvironment network composed of fibroblasts, multipotent stromal cells, blood vessels, immune cells, and secreted factors such as cytokines. Therefore, further studies involving manipulation of the tumor microenvironment could offer an approach to prevent and treat cancer.

Activation of the PI3K/AKT and MEK/ERK pathways is common in different types of cancer (20-23) and, in solid tumors, it is often associated with poor prognosis (24). AKT and ERK play crucial roles in cell proliferation, metastasis, angiogenesis, and drug response. The phosphorylated state of both AKT and ERK is indicative of the activity of tumor signaling $(25,26)$. Moreover, a high expression of both proteins coincides with higher tumor cell viability and proliferation, and lower death in basic experiments, but a poorer prognosis in clinical practice (27). Finally, the PI3K/AKT pathway plays an important role in progression of CRC cells with a BRAF mutation, which can accelerate invasion, migration, and infiltration (28). Here, we treated CRC cells bearing the $\mathrm{BRAF}^{\mathrm{V} 600 \mathrm{E}}$ mutation with vemurafenib and a c-Met inhibitor, individually or in combination. Immunohistochemistry revealed no significant decrease in expression of p-AKT and p-ERK after treatment with vemurafenib alone. However, the c-Met inhibitor synergized with vemurafenib to result in low expression of $\mathrm{P}-\mathrm{AKT}$ and P-ERK. These findings constitute an indirect proof of the impact the combined therapy has in suppressing tumorigenesis and development. Furthermore, it suggests a possible mechanism for cancer cell resistance to vemurafenib and the BRAF inhibitor. Additional in-depth studies are warranted to explore the exact underlying mechanism.

In conclusion, combined treatment with PHA-665752 and vemurafenib suppresses in vitro and in vivo CRC cell growth more effectively than treatment with either agent alone. The targeting of c-Met combined with vemurafenib may represent an effective approach in the management of CRC patients with a BRAF ${ }^{\mathrm{V} 600 \mathrm{E}}$ mutation.

\section{Acknowledgements}

This study was supported by the National Natural Science Foundation of China (no. 81172332).

\section{References}

1. Siegel RL, Miller KD and Jemal A: Cancer statistics, 2015. CA Cancer J Clin 65: 5-29, 2015.

2. Lin PC, Yang YF, Tyan YC, Hsiao ES, Chu PC, Lee CT, Lee JC, Chen YM, Liao PC: Identification of Phosphorylated Cyclin-Dependent Kinase 1 Associated with Colorectal Cancer Survival Using Label-Free Quantitative Analyses. PLoS One 11: e0158844, 2016.

3. Clarke CN and Kopetz ES: BRAF mutant colorectal cancer as a distinct subset of colorectal cancer: Clinical characteristics, clinical behavior, and response to targeted therapies. J Gastrointest Oncol 6: 660-667, 2015

4. Chapman PB, Hauschild A, Robert C, Haanen JB, Ascierto P, Larkin J, Dummer R, Garbe C, Testori A, Maio M, et al: Improved survival with vemurafenib in melanoma with BRAF V600E mutation. N Engl J Med 364: 2507-2516, 2011.

5. Cohen R, Cervera P, Svrcek M, Pellat A, Dreyer C, de Gramont A and André T: BRAF-mutated colorectal cancer: What is the optimal strategy for treatment. Curr Treat Options Oncol 18: 9, 2017.

6. Byeon HK, Na HJ, Yang YJ, Ko S, Yoon SO, Ku M, Yang J, Kim JW, Ban MJ, Kim JH, et al: Acquired resistance to BRAF inhibition induces epithelial-to-mesenchymal transition in BRAF (V600E) mutant thyroid cancer by c-Met-mediated AKT activation. Oncotarget 8: 596-609, 2017.

7. Filitis DC, RauhJ and Mahalingam M: The HGF-cMET signaling pathway in conferring stromal-induced BRAF-inhibitor resistance in melanoma. Melanoma Res 25: 470-478, 2015.

8. Gnad F, Doll S, Song K, Stokes MP, Moffat J, Liu B, Arnott D, Wallin J, Friedman LS, Hatzivassiliou G and Belvin M: Phosphoproteome analysis of the MAPK pathway reveals previously undetected feedback mechanisms. Proteomics 16: 1998-2004, 2016.

9. Barresi V, Bonetti LR and Bettelli S: KRAS, NRAS, BRAF mutations and high counts of poorly differentiated clusters of neoplastic cells in colorectal cancer: Observational analysis of 175 cases. Pathology 47: 551-556, 2015.

10. Gao J, Sun ZW, Li YY and Shen L: Mutations of KRAS and BRAF in Chinese patients with colorectal carcinoma: Analyses of 966 cases. Zhonghua Bing Li Xue Za Zhi 41: 579-583, 2012 (In Chinese).

11. Jang S, Hong M, Shin MK, Kim BC, Shin HS, Yu E, Hong SM, Kim J, Chun SM, Kim TI, et al: KRAS and PIK3CA mutations in colorectal adenocarcinomas correlate with aggressive histological features and behavior. Hum Pathol 65: 21-30, 2017.

12. Chen CT, Kim H, Liska D, Gao S, Christensen JG and Weiser MR: MET activation mediates resistance to lapatinib inhibition of HER2-amplified gastric cancer cells. Mol Cancer Ther 11: 660-669, 2012

13. Joshi M, Rice SJ, Liu X, Miller B and Belani CP: Trametinib with or without vemurafenib in BRAF mutated non-small cell lung cancer. PLoS One 10: e0118210, 2015.

14. Sikkandhar MG, Nedumaran AM, Ravichandar R, Singh S, Santhakumar I, Goh ZC, Mishra S, Archunan G, Gulyás B and Padmanabhan P: Theranostic probes for targeting tumor microenvironment: An overview. Int J Mol Sci 18: pii: E1036, 2017. 
15. Munn DH, Sharma MD, Johnson TS and Rodriguez P: IDO, PTEN-expressing Tregs and control of antigen-presentation in the murine tumor microenvironment. Cancer Immunol Immunother 66: 1049-1058, 2017.

16. Casey SC, Amedei A, Aquilano K, Azmi AS, Benencia F, Bhakta D, Bilsland AE, Boosani CS, Chen S, Ciriolo MR, et al: Cancer prevention and therapy through the modulation of the tumor microenvironment. Semin Cancer Biol 35 (Suppl): S199-S223, 2015.

17. Whitfield JR and Soucek L: Tumo $\mathrm{r}$ microenvironment: Becoming sick of Myc. Cell Mol Life Sci 69: 931-934, 2012.

18. Rakhra K, Bachireddy P, Zabuawala T, Zeiser R, Xu L, Kopelman A, Fan AC, Yang Q, Braunstein L, Crosby E, et al: CD4(+) T cells contribute to the remodeling of the microenvironment required for sustained tumor regression upon oncogene inactivation. Cancer Cell 18: 485-498, 2010.

19. Kenny PA, Lee GY and Bissell MJ: Targeting the tumor microenvironment. Front Biosci 12: 3468-3474, 2007.

20. Kuo YH, Chiang EI, Chao CY, Rodriguez RL, Chou PY, Tsai SY, Pai MH and Tang FY: Dual inhibition of key proliferation signaling pathways in triple-negative breast cancer cells by a novel derivative of taiwanin A. Mol Cancer Ther 16: 480-493, 2017.

21. Mann KM, Ying H, Juan J, Jenkins NA and Copeland NG: KRAS-related proteins in pancreatic cancer. Pharmacol Ther 168: 29-42, 2016.

22. Bresin A, D'Abundo L, Narducci MG, Fiorenza MT, Croce CM, Negrini M and Russo G: TCL1 transgenic mouse model as a tool for the study of therapeutic targets and microenvironment in human B-cell chronic lymphocytic leukemia. Cell Death Dis 7: e2071, 2016.
23. Krpina K, Babarović E, Španjol J, Đorđević G, Maurer T and Jonjić N: Correlation of tumor-associated macrophages and NK cells with bladder cancer size and T stage in patients with solitary low-grade urothelial carcinoma. Wien Klin Wochenschr 128: 248-252, 2016.

24. Ocana A, Vera-Badillo F, Al-Mubarak M, Templeton AJ, Corrales-Sanchez V, Diez-Gonzalez L, Cuenca-Lopez MD, Seruga B, Pandiella A and Amir E: Activation of the $\mathrm{PI} 3 \mathrm{~K} / \mathrm{mTOR} / \mathrm{AKT}$ pathway and survival in solid tumors: Systematic review and meta-analysis. PLoS One 9: e95219, 2014.

25. Baba Y, Tamura T, Satoh Y, Gotou M, Sawada H, Ebara S, Shibuya K, Soeda J and Nakamura K: Panitumumab interaction with TAS-102 leads to combinational anticancer effects via blocking of EGFR-mediated tumor response to trifluridine. Mol Oncol 11: 1065-1077, 2017.

26. Liu L, Liao JZ, He XX and Li PY: The role of autophagy in hepatocellular carcinoma: Friend or foe. Oncotarget 8: 57707-57722, 2017.

27. Wang X, Shi W, Shi H, Lu S, Wang K, Sun C, He J, Jin W, Lv X, Zou H and Shu Y: TRIM11 overexpression promotes proliferation, migration and invasion of lung cancer cells. J Exp Clin Cancer Res 35: 100, 2016.

28. He K, Chen D, Ruan H, Li X, Tong J, Xu X, Zhang L and Yu J: $B R A F$ V600E-dependent Mcl-1 stabilization leads to everolimus resistance in colon cancer cells. Oncotarget 7: 47699-47710, 2016. 